\title{
肘at Second.
}

\section{REVIEWS.}

On the Infuenza, or Epidemic Catarrhal Fever of 1847-48. By Thomas Bevill Peacock, M.D., Licentiate of the Royal College of Physicians, Physician to the Royal Free Hospital, \&c.

THE object of Dr Peacock in publishing the present volume, is to give to the profession a comprehensive account of the recent epidemic, in so far as it fell under his personal observation. In a brief introductory review, he notices, amongst other subjects, the predominating class of diseases and the atmospheric peculiarities which preceded for some time the appearance of the influenza; the difference in time at which it appeared in different parts of the country, and in other countries than our own; the relative number of the community attacked, and the relative fatality of the disease. The numerous synonyms of the affection are then noticed; our author adhering to the name given to it by Huxham, namely Epidemic Catarrhal Fever, although the term Influenza has been so thoroughly sanctioned by general adoption, that he does not altogether discard it.

$\mathrm{He}$ treats the several varieties of the disease under the following heads :

1. Simple catarrhal fever.

2. Catarrhal fever with pulmonary complication; and,

3. Catarrhal fever with predominant disorder of the abdominal organs.

The first and second comprise those to which the term influenza is commonly applied, while the third is subdivided into three divisions, to which we shall presently revert.

The description given by our author of "simple catarrhal fever," is graphic, and extremely accurate. As, however, most of these cases require little medical aid, we proceed at once to the consideration of "catarrhal fever with pulmonary complication."

The author had twenty-eight cases of this nature under his care at the hospital; the local disease taking the form of-

Acute or subacute capillary bronchitis, in eleven cases;

Bronchitis supervening on tuberculous disease of the lungs, in four cases ;

Bronchitis complicated by disease of the heart or aorta, in six cases; and

Pneumonia, in seven cases.

In eight of the eleven cases in which there was acute capillary bronchitis, the disease "commenced suddenly, for the most part with a feeling of cold between the shoulders and down the back, increasing to 
slight shivering or severe rigors, and followed by heat of skin, more or less intense, and in some by perspiration. To these symptoms succeeded pain in the head, chest, and loins; a sense of tightness across the chest; difficulty of breathing, and cough, with usually some soreness of the throat." In two, the disease commenced with sickness and vomiting, combined in one of them with pain in the abdomen.

It is worthy of remark, that seven out of the eleven patients had for some time laboured under other pulmonary affections.

For a detailed account of the symptoms, and the post-mortem appearances in the cases complicated with capillary bronchitis, we must refer our readers to the work itself; we proceed to our author's remarks on the diagnosis of capillary bronchitis, when associated with catarrhal fever.

"In cases of influenza, when an unusual degree of dyspnœea, lividity of the face, and prostration of strength occur, we may with considerable probability conjecture the affection which is about to ensue, while the physical signs remain of an undecided character. It is only, however, at a later period, when effusion to a greater or less extent has already taken place, that the general symptoms and physical signs become sufficiently characteristic to remove all doubt as to the nature of the disease. The only affections with which capillary bronchitis can then be confounded, are the more ordinary forms of bronchitis on the one hand, and pneumonia on the other; and it should be borne in mind, that the disease is generally combined with some degree of both of these affections, and that its character is variously modified by their presence. The following remarks, therefore, only apply to its more characteristic forms :-

"From pneumonia, capillary bronchitis may be distinguished-

" 1 st. By the general symptoms. The less sthenic character of the febrile disturbance, which accompanies all stages of the disease; the greater degree of lividity of the face and extremities, the more rapid prostration of strength, and the earlier appearance of symptoms of asphyxia.

" $2 \mathrm{~d}$. By the physical signs. The absence of dulness on percussion in the earlier stages, and the occurrence of abnormal clearness at the later periods, the fine crepitation generally first audible in the inferior parts of one or both dorsal regions, and then rapidly spreading over all parts of the chest; and the tendency of this sign to pass into the subcrepitant and mucous rhonchus, rather than to give place to evidences of condensation, such as bronchial respiration and increased resonance of the voice and cough.

"3rd. By the peculiar characters of the dyspnoea, cough, and expectoration. The respiratory movements are rapid, short, and hurried, rather than laborious and irregular; and there is a sense of constriction in the chest without pain. The cough comes on generally in paroxysms, and is fatiguing from its frequency, rather than painful or very severe. The sputum consists of small whitishcoloured and viscid pellets, free from air, which have a tendency to become aggregated into tenacious, solid, irregularly shaped masses, and want the russet colour, glairy adhesive quality, and small air-bubbles, which characterise the expectoration in pneumonia.

"From general or ordinary bronchitis, the points of discrimination are less decided ; it will, however, be generally found, that in capillary bronchitis,-

" 1 st. The degree of dyspnœea, and the lividity of the face are greater, and the prostration of strength not only appears earlier, but is throughout more marked.

" 2d. The different forms of crepitant and sibilant rhonchi, are at all times the prevailing physical signs. 
" 3 rd. The cough is less severe, and the sputum in the earlier stages resembles rather aggregations of viscid whitish pellets, than the glairy or muco-purulent masses of ordinary bronchitis.

"Attention to these particulars will sufficiently distinguish capillary bronchitis as a complication of influenza."-Pp.34-5.

We have given the above extract because we believe it to contain the best diagnostic account of capillary bronchitis yet published, and because it will afford our readers a fair idea of the author's power of describing disease as he sees it.

The prognosis in those cases in which capillary bronchitis is associated with influenza is very unfavourable, four of the eleven cases noticed by Dr Peacock having died.

We shall pass without any remarks over our author's observations on the cases in which the local disease took the form of bronchitis, supervening on pulmonary phthisis or complicated with disease of the heart or aorta, and proceed to the subject of influenza complicated with pneumonia. Dr Peacock observes, and our personal experience fully confirms his statement, that the pneumonia occurring in persons suffering from influenza, differed in many respects from ordinary pneumonia. It was attended by an unusual degree of depression of strength, and was combined with various indications of disorder of the chylopoietic viscera, and more or less general bronchial inflammation. The prostration of strength was usually very obvious from nearly the commencement of the attack, before the local affection had gained much ground. The cough was one of the most troublesome symptoms. In three of the seven cases noticed by our author, there was no expectoration, while in the others the sputa presented the mixed character of pneumonia and bronchitis. Neither pain in the chest, nor dyspnœa were very decided, nor were the movements of respiration much accelerated. The pulse was somewhat quicker than usual, but was either soft and compressible, or decidedly small and feeble. The skin was usually cool and moist. The tongue was usually red at the tip and edges, with a whitish-brown fur at the centre and root; it was moist in the early stage, and became drier with the progress of the disease. Anorexia, nausea, and vomiting were early symptoms, to which diarrhœea with abdominal tenderness, and some tympanitis, succeeded. It is worthy of remark that, notwithstanding the threatening character of these cases of pneumonia, they were readily amenable to treatment, and terminated favourably.

Our limited space prevents us from noticing Dr Peacock's third variety-epidemic catarrhal fever with abdominal complication.

We proceed to the division of his work devoted to the treatment of the disease.

In cases of simple catarrhal fever, "the patient was usually directed to be confined to the room or the bed; to take some form of antimonial or diaphoretic medicine; to use the foot bath, and to restrain himself to a low diet. At the end of a day or two, according 
to the urgency of the symptoms, a more nutritious diet was allowed, and some form of tonic or slightly stimulant medicine, with anodynes and expectorants, was prescribed." We very much doubt whether the patient in reality derived any advantage from this treatment. We believe he would have got well just as soon without "antimonial or diaphoretic medicine" or restriction to low diet. Our own experience leads us to the belief, that after having duly regulated the bowels, the most successful treatment consisted in immediate recourse to stimulants and tonics; and that mulled port-wine and beef-tea were of more avail than antimonials or other depressing agents; and the anorexia usually present, rendered any advice regarding spare diet very superfluous. This difference between our author and ourselves is, however, only one of degree, for he concludes by observing, that "tonics and stimulants were employed more freely, and at an earlier period than would, in simple catarrh, have been either necessary or desirable."

The section devoted to the treatment of the cases of influenza with pulmonary complication, is perhaps the most valuable in the whole book, and affords the strongest evidence of the author's skill at the bedside. We strongly recommend it to the most attentive perusal of our readers.

In his remarks on the treatment of influenza with abdominal complication, he specially notices the low rheumatic fever which was frequently present. Under this division he seems to include all those cases in which the pains in the head, back, loins, and extremities were especially severe. Colchicum was his sheet-anchor in these cases; four or five minims of the tincture of the seed, combined with ammonia and opium being given every three, four, or six hours; stimulants or tonics being usually administered at the same time, or later in the attack. Dr Peacock does not make any allusion to a very simple mode of treating the intense evening headache, which has been strongly advocated by Dr Graves, and has proved most efficacious in our own hands, viz., sponging the temples with very hot water. When rheumatic symptoms continued to harass the patient during convalescence, our author's treatment consisted in the internal use of small doses of colchicum, iodide of potassium, and opium, separately or in combination, and in the employment of stimulating and anodyne liniments externally. With regard to the latter, he observes that-

"The most useful external application which I have tried is a combination, in about equal proportions, of the strong tincture of aconite, prepared according to the formula of Dr Fleming,' with the compound camphor and soap liniments. The remedy requires to be rubbed in for a sufficient length

1 As the tinctures of aconite obtained from the London chemists differ extremely in strength, we may observe that $\mathrm{Mr}$ Twinberrow of Edwards Street, Portman Square, and Mr Squire of Oxford Street, prepare the tincture in acoordance with Dr Fleming's formula.-REv. 
of time to produce a strong biting or tingling sensation in the skin, which is succeeded by a general numbness of the surface, that materially alleviates the pain. After having been a few times employed, the proportion of the aconite may be increased to one-half or two-thirds."-P. 96.

We cannot follow Dr Peacock in his inquiry into the nature and causes of the epidemic; but we trust that we have already said enough to ensure for his very admirable and well-timed work a place in the library of all our readers. We took up his volume with very high expectations; we have not been disappointed.

G. E. D.

Fever in its Relations to Sanitary Reform, being the Address in Medicine, delivered at the Sixteenth Anniversary Meeting of the Provincial Medical and Surgical Association, held at Bath, August 16 and 17, 1848. By WM. Davies, M.D., Edin.; Physician to the Bath United Hospital. Pp. 31.

AT the graduation of the University of Edinburgh in 1843, the author of this address obtained the distinguished honour of a gold medal for his dissertation on typhus fever. From the high opinion entertained of his essay by the professors, its publication in a separate form was generally anticipated, and in not doing so, we think $\mathrm{Dr}$ Davies has done himself considerable injustice. An address scarcely admits of that careful sifting of evidence which the nature of his enquiry demanded; so that while the present paper contains a clear announcement of the author's views of fever, it is not so complete in their illustration as is desirable. Dr Davies is himself conscious of this, and has promised to publish an appendix to the next volume of the transactions of the Association, which shall contain such additional materials as he may deem requisite to a full exposition of his subject. We postpone our review of Dr Davies' opinions on fever, until the appearance of this supplement, and confine ourselves at present to a brief outline of the argument in the address.

Dr Davies maintains :-1. That typhus fever is marked by a specific eruption. Without denying that the measly rash may be occasionally absent, we understand Dr Davies' opinion to be, that he would reject all evidence bearing on the origin and dissemination of the disease, derived from cases which did not present this characteristic symptom. 2. That it is propagated by contagion; and, 3. That there is no distinct evidence of its ever spreading otherwise than by contagion. These two propositions embrace the main object of the address, which is to show that defective sanitary arrangements can never in themselves originate genuine typhus, however strongly they may predispose individuals to receive its poison. Dr Davies admits the sufficiency of these causes, more especially when conjoined with poverty and mental distress, to ori- 